\title{
Search for rare processes with DAMA experimental set-ups
}

\author{
A.S. Barabash ${ }^{1}$, P. Belli ${ }^{2}$, R. Bernabei ${ }^{3,2}$, R.S. Boiko ${ }^{4}$, V.B. Brudanin ${ }^{5}$, F. Cappella ${ }^{6, a}$,
} V. Caracciolo ${ }^{7}$, R. Cerulli $^{2}$, D.M. Chernyak ${ }^{4,8}$, C.J. Dai ${ }^{9}$, F.A. Danevich ${ }^{4}$, A. d'Angelo ${ }^{6}$, A. Di Marco ${ }^{2}$, H.L. $\mathrm{He}^{9}$, A. Incicchitti ${ }^{6}$, D.V. Kasperovych ${ }^{4}$, V.V. Kobychev ${ }^{4}$, S.I. Konovalov ${ }^{1}$, H.H. Kuang ${ }^{9}$, X.H. Ma ${ }^{9}$, V. Merlo ${ }^{3}$, F. Montecchia ${ }^{10,2}$, D.V. Poda ${ }^{4,11}$, O.G. Polischuk ${ }^{4}$, X.D. Sheng ${ }^{9}$, V.N. Shlegel ${ }^{12}$, V.I. Tretyak ${ }^{4}$, V.I. Umatov ${ }^{1}$, R.G. Wang ${ }^{9}$, Z.P. Ye ${ }^{9,13}$, and M.M. Zarytskyy ${ }^{4}$

${ }^{1}$ National Research Centre "Kurchatov Institute", Institute of Theoretical and Experimental Physics, 117218 Moscow, Russia

${ }^{2}$ INFN, sez. Roma "Tor Vergata", 00133 Rome, Italy

${ }^{3}$ Dip. di Fisica, Università di Roma "Tor Vergata", 00133 Rome, Italy

${ }^{4}$ Institute for Nuclear Research, 03028 Kyiv, Ukraine

${ }^{5}$ Joint Institute for Nuclear Research, 141980 Dubna, Russia

${ }^{6}$ INFN, sez. Roma, 00185 Rome, Italy

${ }^{7}$ Laboratori Nazionali del Gran Sasso, INFN, 67100 Assergi (AQ), Italy

${ }^{8}$ Kavli Institute for the Physics and Mathematics of the Universe (WPI), University of Tokyo, Kashiwa, 2778583, Japan

${ }^{9}$ Key Laboratory of Particle Astrophysics, Institute of High Energy Physics, Chinese Academy of Sciences, P.O. Box 918/3, Beijing 100049, China

${ }^{10}$ Dip. di Ingegneria Civile e Ingegneria Informatica, Università di Roma "Tor Vergata", 00133 Rome, Italy

${ }^{11}$ CSNSM, Univ. Paris-Sud, CNRS/IN2P3, Université Paris-Saclay, 91405 Orsay, France

${ }^{12}$ Nikolaev Institute of Inorganic Chemistry, 630090 Novosibirsk, Russia

${ }^{13}$ University of Jing Gangshan, Ji'an, Jiangxi, China

\begin{abstract}
Profiting of the favourable conditions offered by the Gran Sasso underground laboratory and of the several low-background DAMA set-ups, many and competitive results have been obtained for rare processes. Here the main results will be briefly resumed and some more details about the latest activities will be given. In particular, the searches on $\beta \beta$ decays of ${ }^{106} \mathrm{Cd}$ and ${ }^{116} \mathrm{Cd}$ and the perspectives of a complementary investigation on those Dark Matter (DM) candidates inducing just nuclear recoils, by exploiting the directionality approach with the anisotropic $\mathrm{ZnWO}_{4}$ scintillators, will be underlined.
\end{abstract}

\section{Introduction}

DAMA (DArk MAtter) is a pioneer project for the investigation of DM, but it is also very active in the search for many other rare processes and in the development of new highly radiopure crystal scintillators. Many important results or competitive limits have also been obtained in the investigation of various rare processes in many experiments performed at the Gran Sasso National Laboratory (LNGS) of the INFN in collaboration with researchers from INR-Kyiv and other institutions.

\footnotetext{
ae-mail: fabio.cappella@roma1.infn.it
} 
The important results obtained in the investigation of DM with the DAMA/NaI and DAMA/LIBRA set-ups are discussed in another article of this volume. Here the main results obtained in the searches for rare nuclear processes with all the DAMA set-ups ${ }^{1}$ will be briefly summarized and some more details about the latest activities will be given: the searches for $\beta \beta$ decays in ${ }^{106} \mathrm{Cd}$ and ${ }^{116} \mathrm{Cd}$ and the perspectives for the investigation of the directionality approach with the $\mathrm{ZnWO}_{4}$ detectors.

\section{Brief review of the main results}

Many important results have been obtained with the DAMA set-ups in experiments studying double beta decay of several candidate isotopes at LNGS. In particular, double beta decay processes of 28 candidate isotopes have been investigated: ${ }^{40} \mathrm{Ca},{ }^{46} \mathrm{Ca},{ }^{48} \mathrm{Ca},{ }^{64} \mathrm{Zn},{ }^{70} \mathrm{Zn},{ }^{100} \mathrm{Mo},{ }^{96} \mathrm{Ru},{ }^{104} \mathrm{Ru},{ }^{106} \mathrm{Cd}$, ${ }^{108} \mathrm{Cd},{ }^{114} \mathrm{Cd},{ }^{116} \mathrm{Cd},{ }^{112} \mathrm{Sn},{ }^{124} \mathrm{Sn},{ }^{134} \mathrm{Xe},{ }^{136} \mathrm{Xe},{ }^{130} \mathrm{Ba},{ }^{136} \mathrm{Ce},{ }^{138} \mathrm{Ce},{ }^{142} \mathrm{Ce},{ }^{156} \mathrm{Dy},{ }^{158} \mathrm{Dy},{ }^{180} \mathrm{~W},{ }^{186} \mathrm{~W}$, ${ }^{184} \mathrm{Os},{ }^{192} \mathrm{Os},{ }^{190} \mathrm{Pt}$ and ${ }^{198} \mathrm{Pt}$. The sensitivities reached on the lifetime of the studied processes are competitive (between $10^{20}$ and $10^{24} \mathrm{yr}$ ), thanks to the radiopurity of the developed detectors and to the used experimental approaches. The obtained results have improved (often by several orders of magnitude) the limits on lifetime obtained by previous experiments and have allowed new observations of two-neutrino double beta decays of ${ }^{100} \mathrm{Mo}$ [1] and ${ }^{116} \mathrm{Cd}$ [2]. Moreover, in the study of decay modes with positron emission or double electron capture, we have obtained ones of the best experimental sensitivities of the field for all the candidate isotopes to these decay modes.

As regards rare $\alpha$ and $\beta$ decays, we have obtained the first observation of the ${ }^{151} \mathrm{Eu} \alpha$ decay with $T_{1 / 2}=5 \times 10^{18}$ yr using a $\mathrm{CaF}_{2}(\mathrm{Eu})$ crystal scintillator [3] and of the $\alpha$ decay of ${ }^{190} \mathrm{Pt}$ to the first excited level $\left(E_{\text {exc }}=137.2 \mathrm{keV}\right)$ of ${ }^{186} \mathrm{Os}$ with $T_{1 / 2}=3 \times 10^{14} \mathrm{yr}$ [4]. Rare $\beta$ decays of ${ }^{113} \mathrm{Cd}$ and ${ }^{48} \mathrm{Ca}$ have been investigated with $\mathrm{CdWO}_{4}[5]$ and $\mathrm{CaF}_{2}(\mathrm{Eu})$ [6] crystal scintillators, respectively.

Pairs of $\mathrm{NaI}(\mathrm{Tl})$ detectors of the DAMA/LIBRA set-up have been used to search for production of correlated $\mathrm{e}^{+} \mathrm{e}^{-}$pairs in the $\alpha$ decay of ${ }^{241} \mathrm{Am}$ [7]. We measured an excess of double coincidences events with energy around $511 \mathrm{keV}$ in faced pairs of detectors that cannot be explained by known side reactions. Therefore, the relative activity for the Internal Pair Production (IPP) with respect to the $\alpha$ decay of ${ }^{241} \mathrm{Am}$ has been determined to be: $(4.70 \pm 0.63) \times 10^{-9}$. This value is of the same order of magnitude as previous determinations and is independent of the livetime estimate.

Solar axions have been searched for studying the conversion of axion to photon (inverse Primakoff effect) in $\mathrm{NaI}(\mathrm{Tl})$ crystals [8] and the resonant absorption of axions emitted in the Sun by ${ }^{7} \mathrm{Li}$ in a $\mathrm{LiF}$ crystal [9] and powders [10].

Delayed coincidences have been investigated to search for exotic particles as Q-balls [11] and SIMPs [12] using the DAMA/NaI detectors and DAEMONs using the specially developed NEMESIS set-up [13].

The electron stability has been investigated searching for electron "disappearance" (i.e. decay into invisible channels as $e^{-} \rightarrow v_{e} \bar{v}_{e} v_{e}$ ) with the DAMA/NaI set-up [14] and with the DAMA/LXe set-up [15] and searching for the $e^{-} \rightarrow v_{e} \gamma$ decay mode with the DAMA/LXe set-up [15, 16].

Finally, competitive limits have been obtained on the lifetime of several other possible nuclear processes. In particular:

- Spontaneous transition of ${ }^{23} \mathrm{Na}$ and ${ }^{127} \mathrm{I}$ nuclei to a superdense state using $\mathrm{NaI}(\mathrm{Tl})$ detectors [17];

- Cluster decays of ${ }^{127} \mathrm{I}$ with $\mathrm{NaI}(\mathrm{Tl})$ detectors [18] and of ${ }^{138} \mathrm{La},{ }^{139} \mathrm{La}$ with $\mathrm{LaCl}_{3}(\mathrm{Ce})$ detector [19];

- Nucleon, di-nucleon and tri-nucleon decay into invisible channels in ${ }^{129} \mathrm{Xe}$ [20] and in ${ }^{136} \mathrm{Xe}$ [21];

\footnotetext{
${ }^{1}$ DAMA operates several low backround set-ups at LNGS, they are: DAMA/LIBRA, DAMA/R\&D, DAMA/CRYS, DAMA/LXe, DAMA/Ge and other HPGe detectors from the STELLA facility.
} 
- Charge non-conserving (CNC) processes in ${ }^{127} \mathrm{I}$ [22], CNC $\beta$ decay of ${ }^{136} \mathrm{Xe}[21],{ }^{100} \mathrm{Mo}$ [1] and ${ }^{139} \mathrm{La} \mathrm{[23],} \mathrm{CNC} \mathrm{electron} \mathrm{capture} \mathrm{with} \mathrm{nuclear} \mathrm{levels} \mathrm{excitation} \mathrm{in}{ }^{127} \mathrm{I}$ and ${ }^{23} \mathrm{Na}$ [24], and in ${ }^{129} \mathrm{Xe}$ [25];

- Nuclear processes violating the Pauli exclusion principle in sodium and in iodine [26, 27];

- Several rare nuclear decays in $\mathrm{BaF}_{2}$ crystal scintillator contaminated by radium [28];

- Long-lived superheavy ekatungsten with radiopure $\mathrm{ZnWO}_{4}$ crystal scintillator [29].

\section{Search for double beta decay in ${ }^{106} \mathrm{Cd}$ with the DAMA/CRYS set-up}

Experimental sensitivities for the search for double beta plus processes (double electrons capture $2 \varepsilon$, electron capture with positron emission $\varepsilon \beta^{+}$and emission of two positrons $2 \beta^{+}$) are substantially more modest with respect to $2 \beta^{-}$processes and only indications exist for the allowed $2 v 2 \varepsilon$ mode in ${ }^{130} \mathrm{Ba}$ $[30,31]$ and ${ }^{78} \mathrm{Kr}[32,33]$ with the half-lives of the order $10^{20}-10^{22} \mathrm{yr}$. At the same time a strong motivation to search for neutrinoless $2 \varepsilon$ and $\varepsilon \beta^{+}$decays are related with the possibility to refine the mechanism of the $0 v 2 \beta^{-}$decay: either it appears due to the Majorana mass of neutrino or due to the contribution of the right-handed admixtures in the weak interaction [34].

The ${ }^{106} \mathrm{Cd}$ isotope is one of the most suitable nuclei to search for the double beta plus processes having a high-energy release in the decay, $Q_{2 \beta}=2775.39(10) \mathrm{keV}$ [35], and relatively high natural isotopic abundance, $\delta=1.245(22) \%$ [36]. In addition the ${ }^{106} \mathrm{Cd}$ is also favored for the possible resonant $0 v 2 \varepsilon$ transitions to excited levels of ${ }^{106} \mathrm{Pd}[37,38]$. The ${ }^{106} \mathrm{Cd}$ is, in fact, one of the most studied nuclei [37].

A new experiment to search for double beta decay in ${ }^{106} \mathrm{Cd}$ is running in the DAMA/CRYS set-up at LNGS using a ${ }^{106} \mathrm{CdWO}_{4}$ crystal scintillator $(215 \mathrm{~g})$, enriched in ${ }^{106} \mathrm{Cd}$ to $66 \%$. It is the third stage of the DAMA activity with this crystal scintillator. In the first stage the ${ }^{106} \mathrm{CdWO}_{4}$ crystal was fixed inside a cavity filled with high purity silicon oil and viewed by two low radioactive photomultiplier tubes (PMT) throughout $\sim 20 \mathrm{~cm}$ long light-guides. After $6590 \mathrm{~h}$ of data taking in the low background DAMA/R\&D set-up, a sensitivity at the level of $T_{1 / 2} \sim 10^{20}-10^{21} \mathrm{yr}$ was reached for different channels of double beta decay of ${ }^{106} \mathrm{Cd}$ [37]. In the second stage, the ${ }^{106} \mathrm{CdWO}_{4}$ crystal was viewed through a lead tungstate $\left(\mathrm{PbWO}_{4}\right)$ crystal light-guide by a low radioactive PMT and installed in the central well of the ultra-low background GeMulti set-up in the STELLA facility at LNGS. This set-up is equipped with four HPGe detectors $\left(225 \mathrm{~cm}^{3}\right.$ each) and was used to search for coincidence events induced, e.g., by the $511 \mathrm{keV}$ gamma(s) produced by the positron annihilations in the $\varepsilon \beta^{+}$or $2 \beta^{+}$ processes. After $13085 \mathrm{~h}$ of data taking, limits on $2 \varepsilon, \varepsilon \beta^{+}$and $2 \beta^{+}$processes in ${ }^{106} \mathrm{Cd}$ were few times improved [39] in comparison with the first stage [37].

The new experiment, now running, was realized in order to increase the detection efficiencies of the coincidence events. For this reason the ${ }^{106} \mathrm{CdWO}_{4}$ has been installed in coincidence with two large volume low background $\mathrm{CdWO}_{4}$ crystal scintillators in close geometry. A scheme of the set-up is depicted in Fig. 1. The ${ }^{106} \mathrm{CdWO}_{4}$ crystal scintillator is in vertical position, viewed through a $\mathrm{PbWO}_{4}$ crystal light-guide by a 3 inches low-radioactive PMT (Hamamatsu R6233MOD). The $\mathrm{PbWO}_{4}$ crystal $(\oslash 40 \times 83 \mathrm{~mm})$ was the one used for the previous stage of the experiment and was developed from deeply purified [40] archaeological lead [41]. The ${ }^{106} \mathrm{CdWO}_{4}$ is almost entirely enclosed by two shaped $\mathrm{CdWO}_{4}$ crystal scintillators. These two crystals are coupled to two low radioactive EMI9265B53/FL PMTs (3 inches diameter) through light-guides made by high purity quartz and polystyrene (both are $66 \mathrm{~mm}$ in diameter and $100 \mathrm{~mm}$ in length). A copper structure keeps the detectors in a fixed position also acting as a shield for the scintillators. The detectors system is installed in the lowbackground DAMA/CRYS set-up. This set-up consists of a passive shield made of high purity copper 


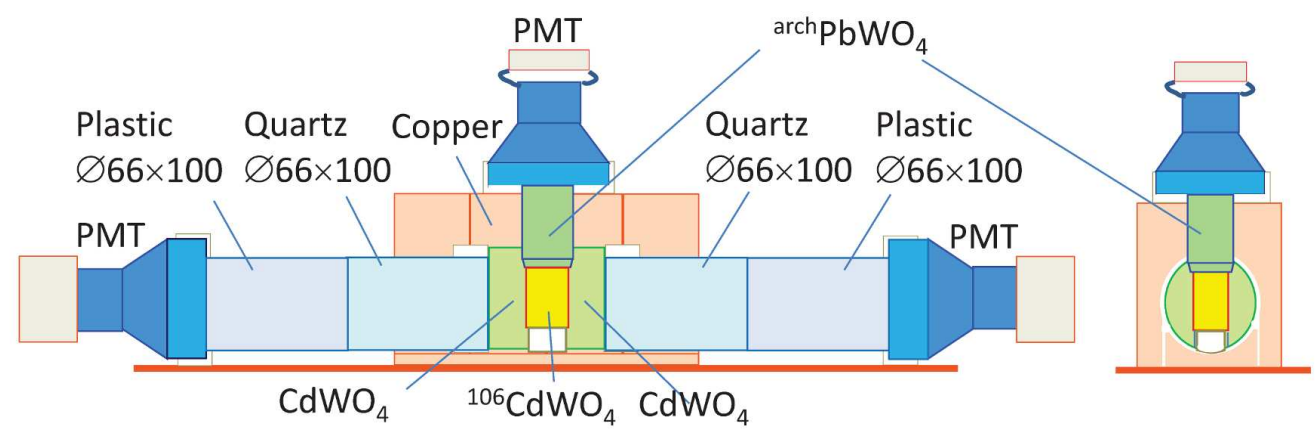

Figure 1. A schematic view of the ${ }^{106} \mathrm{CdWO}_{4}$ set-up installed in the DAMA/CRYS set-up at LNGS.

$(11 \mathrm{~cm})$, lead $(10 \mathrm{~cm})$, cadmium $(2 \mathrm{~mm})$, and polyethylene $(10 \mathrm{~cm})$; it is sealed and continuously flushed by high purity nitrogen gas to prevent the detector to be in contact with the environmental air. In Fig. 2 some pictures of the ${ }^{106} \mathrm{CdWO}_{4}$ set-up during the assembling are shown.

An event-by-event data acquisition system records the amplitude, the arrival time, and the pulse shape of the events by means of a 100 MSamples/s, 14 bit Transient Digitizer DT5724 by CAEN, over a time window of $60 \mu \mathrm{s}$. The low energy part of the ${ }^{106} \mathrm{CdWO}_{4}$ spectrum is dominated by the $\beta$ decay of ${ }^{113} \mathrm{Cd}$ and ${ }^{113 m} \mathrm{Cd}$, not of interest in this measurement. For this reason, the scintillation events of the ${ }^{106} \mathrm{CdWO}_{4}$ with energy below about $500 \mathrm{keV}$ are recorded by the DAQ only if there is a coincidence signal in at least one of the two $\mathrm{CdWO}_{4}$ crystal scintillators. In this way the stored data are considerably reduced. For scintillation events in ${ }^{106} \mathrm{CdWO}_{4}$ with energy above $500 \mathrm{keV}$ the signals of the three detectors are always recorded whether or not a coincidence event is present.
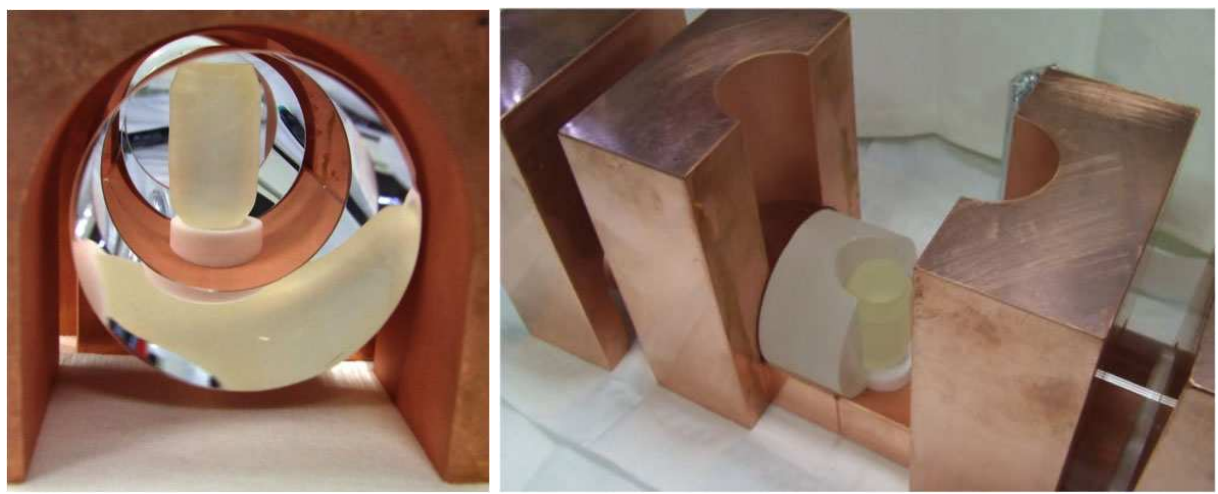

Figure 2. Pictures of the ${ }^{106} \mathrm{CdWO}_{4}$ set-up during the assembling. On the left: the ${ }^{106} \mathrm{CdWO}_{4}$ installed in the central part of the set-up. On the right: one of the $\mathrm{CdWO}_{4}$ enclosing the enriched scintillator is shown together with some copper shaped bricks.

The measurement started in May 2016 and is still in progress. The ${ }^{106} \mathrm{CdWO}_{4}$ and two large $\mathrm{CdWO}_{4}$ scintillators are calibrated with ${ }^{22} \mathrm{Na},{ }^{60} \mathrm{Co},{ }^{133} \mathrm{Ba},{ }^{137} \mathrm{Cs}$ and ${ }^{228} \mathrm{Th} \gamma$ sources. The obtained energy resolution (FWHM) are, e.g.: 16.3(2)\% and 12.9(2)\% for the $662 \mathrm{keV}$ and $1275 \mathrm{keV}$ peaks of the ${ }^{106} \mathrm{CdWO}_{4}$ crystal and $12.4(3) \%$ and $8.3(1) \%$ for the $662 \mathrm{keV}$ and $1275 \mathrm{keV}$ peaks of the $\mathrm{CdWO}_{4}$ detectors, respectively. The ${ }^{22} \mathrm{Na}$ source has also been used to study the coincidence events with energy around $1275 \mathrm{keV}$ in the ${ }^{106} \mathrm{CdWO}_{4}$ crystal and $511 \mathrm{keV}$ in one of the two $\mathrm{CdWO}_{4}$ scintillators. The response of the electronics and DAQ is well in agreement with the expectations. 
The difference in pulse shapes in the $\mathrm{CdWO}_{4}$ scintillators can be used to discriminate $\gamma(\beta)$ events from those induced by $\alpha$ particles. With a preliminary set of data the pulse shape discrimination (PSD) capability of the detectors in the present configuration has been evaluated by using different pulse shape analyses. The separation of the populations of $\alpha$ and $\gamma$ is presently worse than the one obtained in the first stage of the experiment [37]; further analysis is in progress.

With the data collected over $6935 \mathrm{~h}$ a preliminary Time-Amplitude analysis has been performed. With this technique $[42,43]$, by studying the arrival time and the energy of each event, it is possible to tag the fast $\alpha$ decay chain in the ${ }^{232} \mathrm{Th}$ family: ${ }^{224} \mathrm{Ra}\left(Q_{\alpha}=5.79 \mathrm{MeV}, T_{1 / 2}=3.66 \mathrm{~d}\right) \rightarrow{ }^{220} \mathrm{Rn}$ $\left(Q_{\alpha}=6.41 \mathrm{MeV}, T_{1 / 2}=55.6 \mathrm{~s}\right) \rightarrow{ }^{216} \mathrm{Po}\left(Q_{\alpha}=6.91 \mathrm{MeV}, T_{1 / 2}=0.145 \mathrm{~s}\right) \rightarrow{ }^{212} \mathrm{~Pb}$. To select $\alpha$ events in the decay chain the quenching of the scintillation output in the $\mathrm{CdWO}_{4}$ crystal scintillator, the so-called $\alpha / \beta$ ratio, defined as the ratio between the $\alpha$ peak position in the $\gamma$ calibrated scale of a detector and the energy of the alpha particles, has been considered. From this preliminary analysis the contamination of ${ }^{228} \mathrm{Th}$ in ${ }^{106} \mathrm{CdWO}_{4}$ crystal was estimated as: $5(1) \mu \mathrm{Bq} / \mathrm{kg}$.

Considering that for some decay modes the detection efficiencies for coincidence events in the region of interest, evaluated by Monte Carlo simulations, is 4-5 times larger with respect to the previous stage, we expect to improve the experimental sensitivity reaching, for some decay modes in ${ }^{106} \mathrm{Cd}$, the half-life level of $\left(10^{20}-10^{22}\right)$ years. This will allow us to explore for the two neutrino $\epsilon \beta^{+}$decay mode the range of some theoretical predictions.

\section{Search for double beta decay in ${ }^{116} \mathrm{Cd}$ with the AURORA experiment}

The isotope ${ }^{116} \mathrm{Cd}$ is one of the best candidates for $0 v 2 \beta$ experiments thanks to the high Q-value $Q_{2 \beta}=2813.49$ (13) $\mathrm{keV}$ [35], relatively large isotopic abundance $\delta=7.512(54) \%$ [36], possibility of enrichment by ultra-centrifugation in large amount and promising estimations of the decay probability [44-47]. A new search for double beta processes in ${ }^{116} \mathrm{Cd}$ has been carried out by the AURORA experiment, with two ${ }^{116} \mathrm{CdWO}_{4}$ crystal scintillators $\left(580 \mathrm{~g}\right.$ and $582 \mathrm{~g}$ ) enriched in ${ }^{116} \mathrm{Cd}$ to $82 \%$ [48]. Good optical and scintillation properties of the detectors were obtained thanks to the deep purification of ${ }^{116} \mathrm{Cd}$ and $\mathrm{W}$ and the advantage of the low-thermal-gradient Czochralski technique to grow the crystal. The active source approach (high detection efficiency), the low levels of internal contamination in $\mathrm{U}$, Th and $\mathrm{K}$ and the possibility of $\alpha / \beta$ PSD have been exploited to reach the best up-to-date sensitivities in the search for several double beta decay modes of ${ }^{116} \mathrm{Cd}$.

In the AURORA experiment, the two ${ }^{116} \mathrm{CdWO}_{4}$ crystals were installed in the low background DAMA/R\&D set-up at LNGS. The scintillators were fixed inside polytetrafluoroethylene containers filled with ultra-pure liquid scintillator and viewed, through low-radioactive quartz light-guides $(\oslash 7 \times 40 \mathrm{~cm})$, by two 3 inches low radioactive PMTs (Hamamatsu R6233MOD). The passive shield was made of high purity copper $(10 \mathrm{~cm})$, low radioactive lead $(15 \mathrm{~cm})$, cadmium $(1.5 \mathrm{~mm})$ and polyethylene/paraffin (4 to $10 \mathrm{~cm}$ ) to reduce the external background. The whole set-up was contained inside a plexiglas box and continuously flushed by high purity nitrogen gas to remove environmental radon. An event-by-event DAQ system based on a $1 \mathrm{GS} / \mathrm{s} 8$ bit transient digitizer (Acqiris DC270) recorded the amplitude, the arrival time and the pulse shape of the events. The energy scale and resolution of the detector were checked periodically with ${ }^{22} \mathrm{Na},{ }^{60} \mathrm{Co},{ }^{137} \mathrm{Cs},{ }^{133} \mathrm{Ba}$, and ${ }^{228} \mathrm{Th}$ sources. The energy resolution of the ${ }^{116} \mathrm{CdWO}_{4}$ detector for $2615 \mathrm{keV}$ quanta of ${ }^{208} \mathrm{Tl}$ was FWHM $\approx 5 \%$.

The pulse profiles of the events were analyzed by using the optimal filter method $[49,50]$ to discriminate $\gamma(\beta)$ from $\alpha$ events. The PSD was applied to reduce background and to estimate, with the Time-Amplitude analysis [43], the ${ }^{228} \mathrm{Th}$ contamination of the ${ }^{116} \mathrm{CdWO}_{4}$ crystals. The front edge analysis was also used to reject the fast chains of decays ${ }^{212} \mathrm{Bi} \rightarrow{ }^{212} \mathrm{Po}$ from the ${ }^{232} \mathrm{Th}$ family. The ${ }^{116} \mathrm{CdWO}_{4}$ crystal scintillators appeared highly radiopure: $0.022(3) \mathrm{mBq} / \mathrm{kg}$ of ${ }^{228} \mathrm{Th},<0.01 \mathrm{mBq} / \mathrm{kg}$ of ${ }^{226} \mathrm{Ra}, 0.20(1) \mathrm{mBq} / \mathrm{kg}$ of ${ }^{40} \mathrm{~K}$, the total $\alpha$ activity of $\mathrm{U} / \mathrm{Th}$ is $2.3 \mathrm{mBq} / \mathrm{kg}$. 

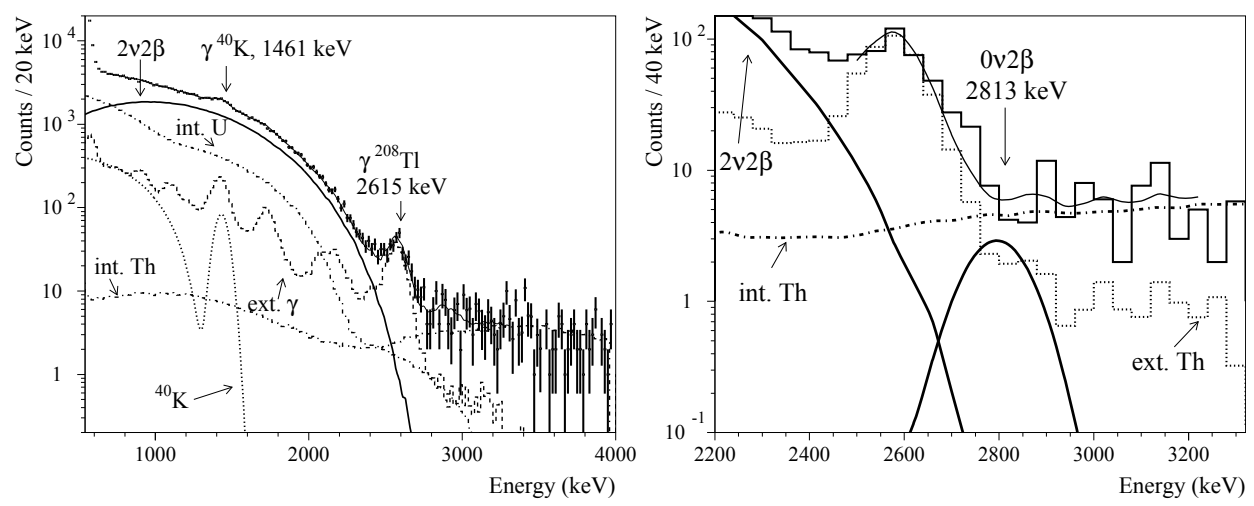

Figure 3. The energy spectrum of $\gamma(\beta)$ events accumulated with the ${ }^{116} \mathrm{CdWO}_{4}$ detectors in the region of interest for $2 v 2 \beta$ decay (on the left, $T=25037 \mathrm{~h}$ ) and $0 v 2 \beta$ decay (on the right, $T=28888 \mathrm{~h}$ ) of ${ }^{116} \mathrm{Cd}$. There are also shown the main components of the background model: the $2 v 2 \beta$ decay of ${ }^{116} \mathrm{Cd}$, the internal contaminations of the ${ }^{116} \mathrm{CdWO}_{4}$ crystals by $\mathrm{U} / \mathrm{Th}, \mathrm{K}$ ("int. U”, "int. Th", “40 K") and contributions from external $\gamma$ quanta ("ext. $\gamma$ " or "ext. Th.”). The peak of the $0 v 2 \beta$ decay of ${ }^{116} \mathrm{Cd}$ excluded at $90 \%$ C.L. is also shown.

The energy spectrum of $\gamma(\beta)$ events selected from the data accumulated over $25037 \mathrm{~h}$ with the ${ }^{116} \mathrm{CdWO}_{4}$ detectors is shown in Fig. 3. The spectrum was fitted in the energy interval $660-3300$ $\mathrm{keV}$ by the model built from the $2 v 2 \beta$ of ${ }^{116} \mathrm{Cd}$, the internal contamination by ${ }^{40} \mathrm{~K},{ }^{232} \mathrm{Th}$ and ${ }^{238} \mathrm{U}$, and the contribution from external $\gamma$ quanta. The model functions were Monte Carlo simulated with the EGS4 package [51], the initial kinematics of the particles emitted in the decays was given by an event generator DECAY0 [52]. The fit gives the half-life of ${ }^{116} \mathrm{Cd}$ relatively to the $2 v 2 \beta$ decay to the ground state of ${ }^{116} \mathrm{Sn}$ as $T_{1 / 2}=[2.69 \pm 0.02$ (stat. $) \pm 0.14($ syst. $\left.)\right] \times 10^{19} \mathrm{yr}$. The main contribution to the systematic error comes from the ambiguity of the radioactive contamination of the ${ }^{116} \mathrm{CdWO}_{4}$ crystals by ${ }^{238} \mathrm{U}$, since the $\beta$ spectrum of ${ }^{234 m} \mathrm{~Pa}$ (daughter of ${ }^{238} \mathrm{U}$ ) competes with the $2 v 2 \beta$ spectrum of ${ }^{116} \mathrm{Cd}$. The obtained result gives the highest up-to-date accuracy for the half-life measurement of the $2 v 2 \beta$ decay of ${ }^{116} \mathrm{Cd}$ (with a signal to background ratio $\simeq 2.6$ in the $1.1-2.8 \mathrm{MeV}$ energy interval).

To estimate a limit on the $0 v 2 \beta$ decay of ${ }^{116} \mathrm{Cd}$ we also included in the analysis the data from the previous stage of the experiment with a similar background counting rate of $\approx 0.1$ counts $/ \mathrm{keV} / \mathrm{kg} / \mathrm{yr}$ in the region of interest (ROI). Furthermore, the background in the ROI was reduced to $\approx 0.07$ counts $/ \mathrm{keV} / \mathrm{kg} / \mathrm{yr}$ by selection of the following chain of decays: ${ }^{212} \mathrm{Bi}\left(Q_{\alpha}=6207 \mathrm{keV}\right) \rightarrow{ }^{208} \mathrm{Tl}$ $\left(Q_{\beta}=4999 \mathrm{keV}, T_{1 / 2}=3.053 \mathrm{~min}\right)$. The obtained energy spectrum was approximated in the energy interval $2.5-3.2 \mathrm{MeV}$ by the background model constructed from the distributions of the $0 v 2 \beta$ (effect searched for) and $2 v 2 \beta$ decays of ${ }^{116} \mathrm{Cd}$, the internal contamination of the crystals by ${ }^{228} \mathrm{Th}$, and the contribution from external $\gamma$ quanta (mainly from the ${ }^{228} \mathrm{Th}$ contamination in the surrounding materials). The fit gives an area of the expected peak $S=-3.2 \pm 10.7$ counts, that is no evidence of the effect. In accordance with ref. [53] 14.6 counts can be excluded at $90 \%$ confidence level (C.L.), that leads to the new limit on the $0 v 2 \beta$ decay of ${ }^{116} \mathrm{Cd}$ to the ground state of ${ }^{116} \mathrm{Sn}: T_{1 / 2}>2.4 \times 10^{23} \mathrm{yr}$. The half-life limit corresponds to the effective neutrino mass limit $\left\langle m_{v}\right\rangle<(1.1-1.6) \mathrm{eV}$, obtained by using the recent nuclear matrix elements reported in refs. [44-47], the phase space factor from ref. [54] and the value of the axial vector coupling constant $g_{A}=1.27$. New improved limits on other $2 \beta$ processes in ${ }^{116} \mathrm{Cd}$ (decays with majoron emission, transitions to excited levels of ${ }^{116} \mathrm{Sn}$ ) were set at the level of $T_{1 / 2}>(0.09-6.3) \times 10^{22} \mathrm{yr}$. 


\section{Exploring the directionality approach with $\mathrm{ZnWO}_{4}$ anisotropic detectors}

In DM direct experiments, in order to point out the presence of a signal of Galactic origin, it is mandatory to pursue a signature for signal identification. The most important one is the model independent DM annual modulation that has been successfully exploited by the DAMA/NaI and DAMA/LIBRA experiments [55]. An independent evidence could be obtained in case of DM candidates inducing just nuclear recoils by pursuing the so-called directionality approach [56]. This strategy is based on the study of the correlation between the arrival direction of the DM particles able to induce nuclear recoils and the Earth motion in the Galactic rest frame. In fact, the solar system moves through the halo of DM and therefore a wind of DM particles apparently flows along a direction opposite to that of solar motion. For an earthly observer the average direction of DM particles changes during the sidereal day because of the rotation of the Earth. Since the directions of the induced nuclear recoils are expected to be strongly correlated with the impinging direction of the DM particles, in principle an experiment able to measure the nuclear track might be suitable to investigate the directionality.

One possibility is to use low pressure gas detector (such as Time Projection Chambers, TPC) where the range of recoiling nuclei is of the order of $\mathrm{mm}$. However, a realistic experiment with low pressure TPC can be limited e.g. by the necessity of an extreme operational stability, of large detector size and of a great spatial resolution in order to reach a significant sensitivity. On the other hand, in solid detectors the range of recoiling nuclei is typically of the order of $\mu \mathrm{m}$. The solution can be offered by anisotropic scintillation detectors $[57,58]$, that does not require the detection and recognition of the track. In these detectors the response for heavy particles depends on the incoming direction of the heavy particles relatively to the crystal axes and the presence of DM induced nuclear recoils can be highlighted by a peculiar variation of the measured counting rate during the sidereal day [59].

\subsection{Advantages of the $\mathrm{ZnWO}_{4}$ anisotropic scintillator}

Recently, measurements and R\&D works have shown that the $\mathrm{ZnWO}_{4}$ scintillators can offer suitable features for a DM experiment based on the directionality. In this crystal the light output and the scintillation decay time for heavy particles ( $p, \alpha$, nuclear recoils) depend on the direction of such particles with respect to the crystal axes while the response to $\gamma / \beta$ radiation is isotropic. In Fig. 4 left the behaviour of the light output ratio for $\alpha$ and $\beta$ ( $\alpha / \beta$ ratio) is reported as a function of the energy and of the direction of the $\alpha$ beam in a $\mathrm{ZnWO}_{4}$ crystal [60]. The response of the detector for
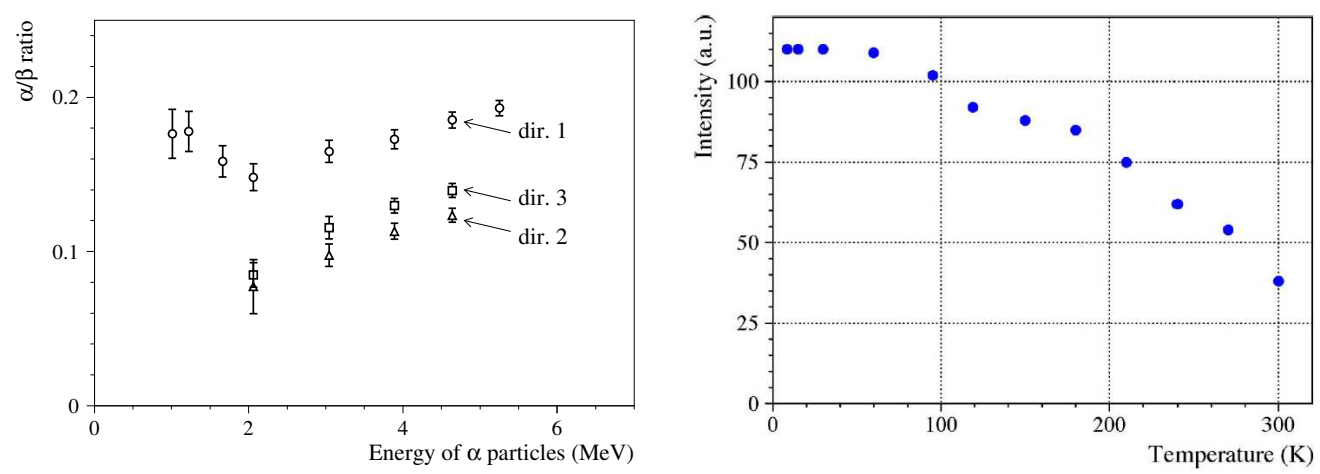

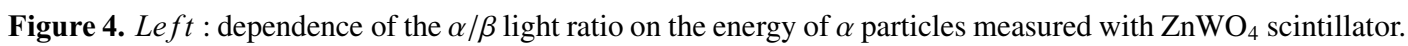
The crystal was irradiated in the directions perpendicular to (010), (001) and (100) crystal planes (directions 1 , 2 and 3, respectively). The anisotropic behaviour of the crystal is evident [60]. Right : dependence of the light output of the $\mathrm{ZnWO}_{4}$ as a function of the temperature, for excitation with ${ }^{241}$ Am $\alpha$ particles [61]. 
$\alpha$ particles measured along direction 1 is about 1.5 times larger than that measured along direction 2, and about 1.4 times larger than that measured along direction 3. On the contrary, the anisotropy of the light response of the $\mathrm{ZnWO}_{4}$ scintillator disappears in case of electron excitation. The same behaviour has been reported also for the anthracene in Ref. [57, 62]. Measurements with a neutron beam to study the anisotropy of the $\mathrm{ZnWO}_{4}$ crystal for recoils at keV energy range are now under way with a neutron generator producing $14 \mathrm{MeV}$ neutrons at ENEA-CASACCIA [63].

In addition to the anisotropy, the recently developed $\mathrm{ZnWO}_{4}$ scintillators have also other important characteristics for a DM experiment: i) very good level of radiopurity [64]; ii) high light output that is to say energy threshold at level of few keV feasible [65]; iii) high stability of the running conditions; iv) sensitivity to both small and large mass DM candidates inducing nuclear recoils thanks to three target nuclei with very different masses; v) possibility to realize crystals with masses of some $\mathrm{kg}$ [66].

As regards the measured radioactive contamination in radiopure $\mathrm{ZnWO}_{4}$ scintillators, it is: < $0.002 \mathrm{mBq} / \mathrm{kg}$ for ${ }^{228} \mathrm{Th}$ and ${ }^{226} \mathrm{Ra}\left(\sim 0.5 \mathrm{ppt}\right.$ for ${ }^{232} \mathrm{Th}$ and $\sim 0.2 \mathrm{ppt}$ for ${ }^{238} \mathrm{U}$, assuming the secular equilibrium of the ${ }^{232} \mathrm{Th}$ and ${ }^{238} \mathrm{U}$ chains), $<0.02 \mathrm{mBq} / \mathrm{kg}$ for ${ }^{40} \mathrm{~K}$; the total $\alpha$ activity is $0.18 \mathrm{mBq} / \mathrm{kg}$ [64]. Moreover, for $\mathrm{ZnWO}_{4}$ the shape of the scintillation pulse depends on the type of irradiation [60]; this feature allows one to discriminate $\gamma(\beta)$ events from those induced by $\alpha$ particles. This PSD capability is of general interest.

Another feature of this scintillator is the relatively high light output $\approx 13-20 \%$ of $\mathrm{Na}(\mathrm{Tl})$. It has been observed that the light output largely increases when the crystal scintillator working temperature is decreased [61] (see Fig. 4 right). A cryostat for low temperature measurement has been recently realized at LNGS for the purpose to determine the behaviour and the low energy threshold reachable for $\mathrm{ZnWO}_{4}$ detectors when operated at low temperature.

\subsection{The expected signal for a directionality experiment with anisotropic detectors}

Taking into account the mean DM arrival directions during the sidereal day ${ }^{2}$, it is very convenient to consider a directionality experiment performed at the LNGS latitude: $42^{\circ} 27^{\prime} \mathrm{N}$ (see Fig. 5 left). In fact, in this case, at $21 \mathrm{~h} \mathrm{LST}$ the DM particles come mainly from the top, while $12 \mathrm{~h}$ later they come from the North and parallel to the horizon line. Thus, the optimal performance for an anisotropic
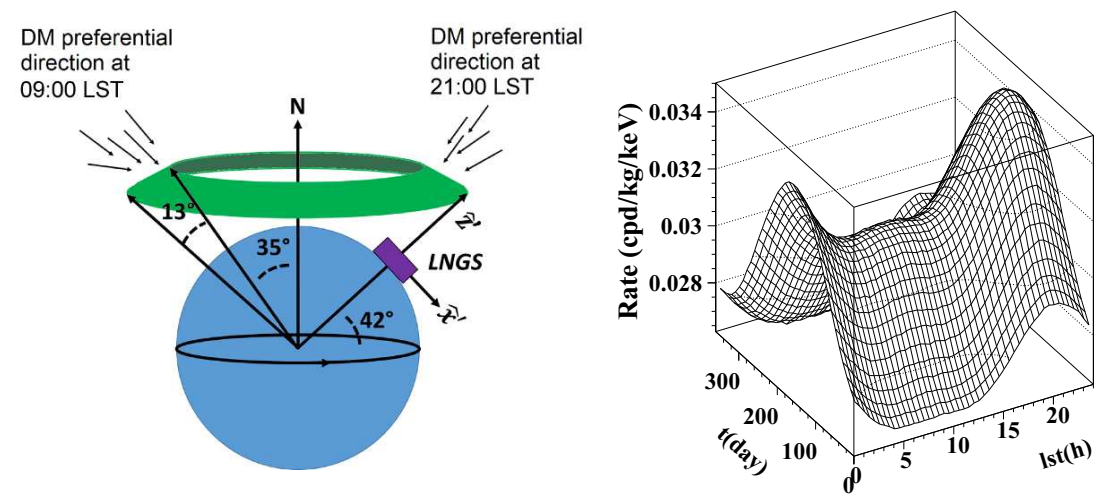

Figure 5. Left : Area in the sky from which DM particles are preferentially expected. Right : time variation of the $[2,3] \mathrm{keV}$ rate expected for $\mathrm{ZnWO}_{4}$ detector when considering the DM scenario given in the text.

\footnotetext{
${ }^{2}$ In the horizontal coordinate system located at the North Pole, the altitude of the mean DM arrival direction experiences a slight annual variation around $48^{\circ}$, due to the Earth revolution velocity, while the azimuth angle has a diurnal variation due to the Earth rotation.
} 
detector is obtained when arranging the crystal axis that corresponds to the largest light output in the vertical direction and the axis that gives the smallest light output towards the North. With this configuration the range of variability of the anisotropic detector response during a sidereal day is at maximum. As a consequence of time-varying detector response, the counting rate measured in a fixed low energy interval by an anisotropic scintillator is expected to vary during a sidereal day. As an example, Fig. 5 right shows the effect achievable in the [2-3] keV energy window with a $\mathrm{ZnWO}_{4}$ detector when considering a DM candidate with spin independent interaction, mass $10 \mathrm{GeV}$ and cross section on nucleon $\sigma_{p}=5 \times 10^{-5} \mathrm{pb}$. We also assume isothermal halo model, Maxwellian velocity distribution, $0.3 \mathrm{GeV} / \mathrm{cm}^{3}$ local density and the same form factor, scaling law, energy resolution and detector response to $\mathrm{Zn}, \mathrm{W}$ and $\mathrm{O}$ recoils as that considered in ref. [59]. The absolute maximum rate is at day 152 and at $21 \mathrm{~h} \mathrm{LST}$ when the DM flux is at maximum and the DM preferential arrival direction is near the zenith, that is near the crystal axis with the largest light output.

\section{Conclusions}

The main results obtained with DAMA experimental set-ups in the search for rare processes have been briefly resumed. Some further details have been given about the latest activities: the searches on $\beta \beta$ decays of ${ }^{106} \mathrm{Cd}$ and ${ }^{116} \mathrm{Cd}$ with enriched $\mathrm{CdWO}_{4}$ scintillators and the feasibility study for a possible future investigation on $\mathrm{DM}$ with the directionality approach using anisotropic $\mathrm{ZnWO}_{4}$ scintillators.

\section{References}

[1] P. Belli et al., Nucl. Phys. A 846, 143 (2010).

[2] O.G. Polischuk et al., AIP Conf. Proc. 1894, 020018 (2017).

[3] P. Belli et al., Nucl. Phys. A 789, 15 (2007).

[4] P. Belli et al., Phys. Rev. C 83, 034603 (2011).

[5] P. Belli et al., Phys. Rev. C 76, 064603 (2007).

[6] R. Bernabei et al., Nucl. Phys. A 705, 29 (2002).

[7] R. Bernabei et al., Eur. Phys. J. A 49, 64 (2013).

[8] R. Bernabei et al., Phys. Lett. B 515, 6 (2001).

[9] P. Belli et al., Phys. Lett. B 711, 41 (2012).

[10] P. Belli et al., Nucl. Phys. A 806, 388 (2008).

[11] F. Cappella et al., Eur. Phys. J. direct C 14, 16 (2002).

[12] R. Bernabei et al., Phys. Rev. Lett. 83, 4918 (1999).

[13] R. Bernabei et al., Mod. Phys. Lett. A 27, 1250031 (2012).

[14] P. Belli et al., Phys. Lett. B 460, 236 (1999).

[15] P. Belli et al., Astrop. Phys. 5, 217 (1996).

[16] P. Belli et al., Phys. Rev. D 61, 117301 (2000).

[17] R. Bernabei et al., Eur. Phys. J. A 23, 7 (2005).

[18] R. Bernabei et al., Eur. Phys. J. A 24, 51 (2005).

[19] R. Bernabei et al., Nucl. Instr. Meth. A 555, 270 (2005).

[20] R. Bernabei et al., Phys. Lett. B 493, 12 (2000).

[21] R. Bernabei et al., Eur. Phys. J. A 27 s01, 35 (2006).

[22] R. Bernabei et al., Eur. Phys. J. C 72, 1920 (2012).

[23] R. Bernabei et al., Ukr. J. Phys. 51, 1037 (2006). 
[24] P. Belli et al., Phys. Rev. C 60, 065501 (1999).

[25] P. Belli et al., Phys. Lett. B 465, 315 (1999).

[26] R. Bernabei et al., Eur. Phys. J. C 62, 327 (2009).

[27] R. Bernabei et al., Phys. Lett. B 408, 439 (1997).

[28] P. Belli et al., Eur. Phys. J. A 50, 134 (2014).

[29] P. Belli et al., Phys. Scr. 90, 085301 (2015).

[30] A.P. Meshik et al., Phys. Rev. C 64, 035205 (2001).

[31] M. Pujol et al., Geochim. Cosmochim. Acta 73, 6834 (2009).

[32] Yu.M. Gavrilyuk et al., Phys. Rev. C 87, 035501 (2013).

[33] S.S. Ratkevich et al., arXiv:1707.07171 [nucl-ex].

[34] M. Hirsch et al., Z. Phys. A 347, 151 (1994).

[35] M. Wang et al., Chin. Phys. C 41, 030003 (2017).

[36] J. Meija et al., Pure Appl. Chem. 88, 293 (2016).

[37] P. Belli et al., Phys. Rev. C 85, 044610 (2012).

[38] M.I. Krivoruchenko et al., Nucl. Phys. A 859, 140 (2011).

[39] P. Belli et al., Phys. Rev. C 93, 045502 (2016).

[40] R.S. Boiko et al., Inorganic Materials 47, 645 (2011).

[41] F.A. Danevich et al., Nucl. Instr. Meth. A 603, 328 (2009).

[42] F.A. Danevich et al., Phys. Lett. B 344, 72 (1995).

[43] F.A. Danevich et al., Nucl. Phys. A 694, 375 (2001).

[44] T.R. Rodryguez, G. Martynez-Pinedo, Phys. Rev. Lett. 105, 252503 (2010).

[45] F. Simkovic, V. Rodin, A. Faessler, P. Vogel, Phys. Rev. C 87, 045501 (2013).

[46] J. Hyvarinen, J. Suhonen, Phys. Rev. C 91, 024613 (2015).

[47] J. Barea, J. Kotila, F. Iachello, Phys. Rev. C 91, 034304 (2015).

[48] A.S. Barabash et al., JINST 6, P08011 (2011).

[49] E. Gatti, F. De Martini, Nuclear Electronics II (IAEA, Vienna, 1962) 265.

[50] L. Bardelli et al., Nucl. Instr. Meth. A 569, 743 (2006).

[51] W.R. Nelson et al., SLAC-Report-265, Stanford, 1985.

[52] O.A. Ponkratenko et al., Phys. At. Nucl. 63, 1282 (2000).

[53] G.J. Feldman, R.D. Cousins, Phys. Rev. D 57, 3873 (1998).

[54] J. Kotila, F. Iachello, Phys. Rev. C 85, 034316 (2012).

[55] R. Bernabei et al., Eur. Phys. J. C 73, 2648 (2013).

[56] D.N. Spergel, Phys. Rev. D 37, 1353 (1988).

[57] P. Belli et al., Nuovo Cimento C 15, 475 (1992).

[58] R. Bernabei et al., Eur. Phys. J. C 28, 203 (2003).

[59] F. Cappella et al., Eur. Phys. J. C 73, 2276 (2013).

[60] F.A. Danevich et al., Nucl. Instrum. Meth. A 544, 553 (2005).

[61] L.L. Nagornaya et al., IEEE Trans. Nucl. Sci. 56, 994 (2009).

[62] P. Schuster, E. Brubaker, IEEE Trans. Nucl. Sci. 63, 1942 (2016).

[63] C. Taruggi, ROM2F/2016/06.

[64] P. Belli et al., Nucl. Instrum. Meth. A 626-627, 31 (2011).

[65] P. Belli et al., J. Phys. G 38, 115107 (2011).

[66] E.N. Galashov et al., Functional Materials 16, 63 (2009). 